\title{
Atomistic evaluation of the stress concentration factor of graphene sheets having circular holes
}

\author{
S.K. Jalali ${ }^{a, *}$, M.J. Beigrezaee ${ }^{a}$, N.M. Pugno ${ }^{b, c, d}$ \\ a Department of Mechanical Engineering, Kermanshah University of Technology, Kermanshah, Iran \\ ${ }^{b}$ Laboratory of Bio-Inspired \& Graphene Nanomechanics, Department of Civil, Environmental and Mechanical Engineering, \\ Università di Trento, via Mesiano, 77, I-38123 Trento, Italy \\ ${ }^{c}$ Ket Lab, Edoardo Amaldi Foundation, Italian Space Agency via del Politecnico snc, I-00133 Roma, Italy \\ d School of Engineering \& Materials Science, Queen Mary University of London, Mile End Road, London E1 4NS, UK
}

\begin{abstract}
Stress concentration factor concept has been developed for single-layered graphene sheets (SLGSs) with circular holes through an atomistic point of view by the application of molecular structural mechanics (MSM) approach. In this approach the response of SLGSs against unidirectional tensile loading is matched to the response of a frame-like macro structure containing beam elements by making an equivalence between strain energies of beam elements in MSM and potential energies of chemical bonds of SLGSs. Both chirality and size effects are considered and the atomistic evaluation of stress concentration factor is performed for different sizes of circular holes. Also, molecular dynamics simulations are implemented to verify the existence and location of the predicted stress concentration. The results reveal that size effects and the diameters of circular holes have a significant influence on the stress concentration factor of SLGSs and armchair SLGSs show a larger value of stress concentration than zigzag ones.
\end{abstract}

\section{Keywords}

Molecular Structural Mechanics (MSM), Defected graphene, Stress concentration factor, Circular holes

\section{Introduction}

Graphene, thanks to its all-surface nature, has been considered as a main candidate for overcoming future technology challenges in nanotechnology. The potential application of graphene is extensively investigated in various domains like health and environment, electronic devices, spintronics, photonics and optoelectronics, sensors and actuators, flexible electronics, energy storage and conversion, nanocomposites, and biomedical applications [1]. Because of the wide application of graphene in nanotechnology, theoretical and experimental evaluations of its unique mechanical properties have been of great interest [2-4]. Besides, the mechanical behaviors of graphene under different loading conditions, such as vibration, bending, buckling and fatigue, have been studied [5-8]. Implementation of continuum mechanics in order to analyze the behavior of nanostructures bearing mechanical loads has received a notable attraction owing to its computational advantages; however, due to elimination of structural discreteness the obtained results are not realistic enough. To overcome this limitation, an atomistic point of view needs to be adopted to take into account the discrete nature of nanostructures. In spite of successful implementation of computational physics methods, i.e. ab initio [9], Monte Carlo simulations [10], and molecular dynamics (MD) simulations [11], the computational cost has always been a noteworthy deficiency. Accordingly, many attempts have been conducted to find semi-atomistic methods which include not only the computational advantages and simplicity of the continuum view, but also the realistic standpoint of atomistic approaches. $\mathrm{Li}$ and Chou 
introduced Molecular structural mechanics (MSM) as a semi-atomistic approach by establishing a linkage between structural and molecular mechanics [12]. The concept of this approach is to consider a nanostructure as a frame-like structure and the chemical bonds between two nearest-neighboring atoms acting like structural members. MSM has attracted the attention in the field of nanostructures and many researchers have applied this approach for investigating the behavior of nanostructures and some modifications are proposed to improve its versatility for special cases of study. Using MSM, Sakhaee-Pour et al. [13] studied the potential application of defect-free single-layered graphene sheets (SLGSs) as mass sensors for detection of atomistic dusts. Both zigzag and armchair configurations with cantilever or bridge boundary conditions were considered. The results revealed that the frequency of graphene was highly sensitive to an added mass of the order of 10-6 fg. Vibrational analysis of carbon nanotubes and graphene sheets by using MSM approach was conducted by Hashemnia et al. [14]. They certified that the vibration frequencies obtained by MSM were in good agreement with the literature. A study on the elastic buckling of SLGSs was done by Sakhaee-Pour [15]. The elastic buckling forces of the cantilever and bridge zigzag and armchair SLGSs with different side lengths and aspect ratios were calculated. The atomistic simulation results based on MSM were also used to develop predictive equations via a statistical nonlinear regression model. Wang et al. [16] evaluated the influence of Stone-Wales defects on elastic properties of graphene nanofilms applying MSM based finite element method. They considered several possible influencing factors, including the number and types of Stone-Wales defects, the distance between two defects and the position of defects in the graphene, to evaluate Young's moduli and Poisson's ratios of armchair and zigzag monolayer graphene nanofilms. Wang et al. [17] analyzed vibration characteristics of wrinkled SLGSs. Based on the MSM simulation results and the continuum thin plate model, a prediction model was proposed to obtain the natural frequency of wrinkled SLGS. Firouz-Abadi et al. [18] proposed a modified MSM model to improve the accuracy of this approach for the analysis of transverse deformations. To this aim, a sample SLGS under a uniform pressure was modeled by both MD and MSM methods. The sectional properties of the beam element of MSM were modified such that the difference between the results of the molecular mechanic's model and the molecular dynamics simulation was minimized. Using this modified model, the buckling behavior of graphene under a uniform edge pressure was investigated subjected to different boundary conditions for both zigzag and armchair chirality. Surveying the literature reveals that MSM has been effectively implemented by the researchers to study the mechanical properties and characteristics of nanostructures and specially carbon nanostructures, i.e. SWCNTs and SLGSs. Carbon atoms of graphene are ideally arranged on a perfect hexagonal lattice. However, it is reported experimentally [19] and theoretically [20] that inevitable defects exist that occur in graphene structure during its fabrication process which make significant drastic changes in its physical, mechanical, and electrical properties [21]. Missing carbon atoms from the hexagonal lattice known as vacancies is a common defect in graphene sheets. Vacancies, having diverse sizes, shapes, and distributions, have been extensively examined in the literature in order to investigate their influence on the mechanical characterization of graphene sheets [22-28]. In classical structural engineering, the influence of geometrical discontinuities like holes, cavities and cracks is taken into account by introducing the concept of stress concentration [29]. This concept is employed by designers to account for the localized increase in stress at a point with geometrical sudden changes, with the nominal stress being multiplied by a stress concentration factor to obtain an estimate of the local stress at the critical point [30]. Recently, the production of largescale graphene sheets is reported [31] which can be considered as a herald of wide application of this nanostructure in future nanotechnology products. Hence, as a mechanical planar structural member, an engineering point of view to graphene sheets may be valuable [25$28,32]$. In this way, the graphene sheets with circular-shaped vacancies may be considered as panels having circular holes. The present work aims to develop the classical concept of stress concentration factor for studying the influence of vacancies on the failure analysis of graphene sheets under tensile load conditions. To take into account the discrete nature of SLGSs, the MSM approach is adopted and the stress concentration factor is calculated and compared with the stress concentration factor predicted by classical elasticity for thin panel with circular holes. 


\section{Definition of stress concentration factor}

In classical elasticity, the presence of sudden changes in the geometry of cross section results in local modification of the stress distribution. A localized high stress is known as stress concentration, measured by the stress concentration factor, $\mathrm{K}$, which is defined as the ratio of the peak stress, omax, to a reference stress in the body, oref:

$$
K=\frac{\sigma_{\max }}{\sigma_{\text {ref }}}
$$

The most well-known case of a sudden change in the geometry is the existence of holes in structures. Consider a thin panel of finite width $h$, and thickness $t$, containing a single circular hole of diameter $d$, subjected to an uniaxial tension stress, $\sigma$, caused by an applied load, P (See Fig. 1). Based on the classical theory of elasticity [29], the maximum stress, omax, occurs at point $M$. There are two options for reference stress, oref , corresponding to two ways of definition of the cross sectional area.

Option one: The nominal area, also named as gross crosssectional area, is considered and the associated stress concentration factor $\mathrm{Kg}$ is defined as:

$$
K_{g}=\frac{\sigma_{\max }}{\sigma}, \quad \sigma=\frac{P}{h t}
$$

Option two: The effective area, also named as net cross-sectional area, is considered and the associated stress concentration factor $\mathrm{Kn}$ is defined as:

$$
K_{n}=\frac{\sigma_{\max }}{\sigma_{n}}, \quad \sigma_{n}=\frac{P}{(h-d) t}
$$

Using Eq. (2) and Eq. (3), one can easily obtain the relationship between gross and net stress concentration factors as follows:

$$
K_{n}=K_{g}\left(1-\frac{d}{h}\right)
$$

Although calculation of the maximum stress can be performed by either $\mathrm{Kn}$ or $\mathrm{Kg}$, in the present work, the gross factor, $\mathrm{Kg}$, is considered. Theoretical approaches, like theory of elasticity and numerical methods as well as experimental ones like photo elasticity, are widely implemented to determine the stress concentration factor. An analytical solution for a panel of finite width having a single circular hole, as shown in Fig. 1 is reported in [29] as follows:

$$
\begin{aligned}
& K_{g}=0.284+\frac{2}{1-\alpha}-0.6(1-\alpha)+1.32(1-\alpha)^{2}, \\
& \alpha=d / h
\end{aligned}
$$

where $\alpha$ is the dimensionless hole parameter, which indicates the relative size of the circular hole with respect to the width of the panel.

\section{Atomistic simulation of SLGSs}

\subsection{MSM approach for SLGSs}

The MSM approach [12] is implemented for atomistic evaluation of stress concentration factor of SLGSs having circular-shaped vacancies. The main idea of MSM is to match the response of a nanostructure against 
a load condition to the response of a frame-like macro structure containing beam and bar elements. The geometry of frame-like structure is constructed similar to the lattice arrangement of the associated nanostructure. The key point for matching the response is to make a coequality between strain energies of structural element in MSM and potential energies of chemical bonds. Implementing this energy equivalency, one can obtain the stiffness and cross section of the equivalent structural element. For the present SLGSs having circular holes, a frame-like structure composed of connecting beams similar to hexagonal lattice of carbon atoms is defined. The length of beams, $L$, is considered equals to the carbon-carbon covalent bond

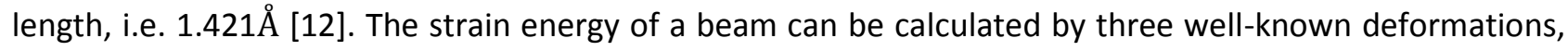
i.e. stretching energy, $\mathrm{Ur}$, bending energy, $\mathrm{U} \theta$, and torsional energy, , $\mathrm{U} \phi$ as follows:

$$
\begin{aligned}
& U_{r}=\frac{E A}{2 L}(\Delta r)^{2}, \\
& U_{\theta}=\frac{E I}{2 L}(\Delta \theta)^{2}, \\
& U_{\varphi}=\frac{G J}{2 L}(\Delta \varphi)^{2}
\end{aligned}
$$

where $\mathrm{E}$ and $\mathrm{G}$ are the elastic and shear modulus, $\mathrm{A}, \mathrm{I}$, and $\mathrm{J}$ are the area, the moment of inertia, and the polar moment of inertia of the cross section of the equivalent beam, respectively. Also, $\Delta r, \Delta \theta$, and $\Delta \phi$ are the stretching displacement and bending and torsional rotations, respectively. The potential energy of covalent bond for carbon atoms in the hexagonal lattice of SLGSs can be evaluated as follows [12]:

$$
\begin{aligned}
& U_{r}=0.5 k_{r}(\Delta r)^{2}, \\
& U_{\theta}=0.5 k_{\theta}(\Delta \theta)^{2}, \\
& U_{\varphi}=0.5 k_{\varphi}(\Delta \varphi)^{2}
\end{aligned}
$$

By equating Eq. (6) and Eq. (7) one may obtain the tensile rigidity, $E A \mathrm{~kL}=r$, the flexural rigidity, $\mathrm{El} \mathrm{kL}=\theta$, and the torsional rigidity, $\mathrm{GJ} \mathrm{kL}=\phi$. Also, considering a circular cross section, the elastic modulus, $\mathrm{E}$, the shear modulus, $\mathrm{G}$, and the diameter $\mathrm{dc}$ of the equivalent beam can be obtained as:

$$
d_{c}=4 \sqrt{k_{\theta} / k_{r}}, \quad E=\frac{k_{r}^{2} L}{4 \pi k_{\theta}}, \quad G=\frac{k_{r}^{2} k_{\varphi} L}{8 \pi k_{\theta}^{2}}
$$

In the present study, among computational approaches in structural mechanics for stress analysis, finite element method (FEM), which has been suggested by various researchers, is selected. It should be noted that as attributing material properties to carbon chemical bonds may not make a physical sense and cause negative bulk modulus in FEM analysis, the tensile, the flexural, and the torsional rigidity are directly used to define the mechanical properties of the beam elements. In MSM model of SLGS, a 2D hexagonal lattice of equivalent beams corresponding to SLGS dimensions is constructed. Each covalent bond is discretized using three linearly interpolated beam elements. In order to form a circular shaped hole, a circle whose center coordinate matches the coordinate of nearest carbon atom to the center of SLGS is defined and all the atoms located inside the circle are removed. The reported radius for holes is the radius of this cutting circle. It is noted that due to discrete nature of hexagonal lattice, increasing the radius may not change the size of holes until some other atoms come to the circle. Therefore, in the steps of increasing the radius, the maximum radius which causes a change in the size of hole is reported. Dangling bonds are kept. For nodes located at the left edges, the movements along $x$ direction and rotations are fixed, while, upper and lower edges are allowed to be free. The loading is applied in form of concentrated nodal force which is equally dispersed at the nodes of the right edge. It is noted that the value of nodal force is not the matter as the solution is linear under the proportional limit. The maximum and average stresses are proportionally related to the applied force and consequently the stress concentration factor is not affected by the value of force. The forcedisplacement equation of an equivalent beam is introduced as: 


$$
\left[K_{i}\right]\left\{u_{i}\right\}=\left\{f_{i}\right\}
$$

in which, [ ] Ki , \{ui\}, and $\{f\} \mathrm{i}$ are respectively the stiffness matrix, the nodal displacement vector, and the nodal force vector of the beam element. The component of [ ] Ki can be calculated in terms of stiffness and cross section parameters of equivalent beam [12]. The governing differential equations of motion for the entire system of the frame-like structure can be constructed by assembling the local element matrices as follows:

$$
[K]\{u\}=\{f\}
$$

in which, [ ] $\mathrm{K}$ is the global stiffness matrix, $\{\mathrm{u}\}$ is the global nodal displacement vector, and $\{f\}$ is the nodal external force vector. By solving Eq. (10), one can calculate the displacements, strains and stresses in a SLGSs to calculate the stress concentration factor. Then, based on Eq. (2) the gross stress concentration factor, $\mathrm{Kg}$, can be calculated by dividing the maximum axial stress of beam elements to the average stress far from the circular boundaries (Fig. 2).

\subsection{MD simulation for SLGSs}

MD simulation has been performed by means of the open source MD simulator, i.e. large-scale atomic/molecular massively parallel simulator (LAMMPS), using a velocity-Verlet algorithm with a time step of $0.5 \mathrm{fs}$, in order to make an insight to the influence of circular holes on the tensile strength of SLGSs. The adaptive intermolecular reactive empirical bond order potential (AIREBO) [33] through the Nose-Hoover thermostat [34] is applied. As the present MSM model does not take into account thermal effects, MD simulations are also carried out at low temperature conditions, $1 \mathrm{~K}$, to avoid thermal fluctuations. Dangling bonds are also kept in MD simulations. Boundary conditions are implemented by fixing motion of one layer of carbon atoms along $x$ direction on the left edges of SLGSs and a stepwise strain is applied incrementally.

\section{Results and discussion}

Atomistic evaluation of stress concentration factor, $\mathrm{Kg}$, is performed by MSM. The introduced force constants in Eq. (7) are considered as [12]: $\mathrm{kr}=6.52 \mathrm{Nnm} \mathrm{E}-7-1, \mathrm{k} \theta=8.76 \mathrm{Nnm} \mathrm{E}-10, \mathrm{k} \phi=2.78 \mathrm{Nn} \mathrm{E}-10 \mathrm{~m}$. To take into account the chirality effects, SLGSs with either armchair or zigzag configurations are considered. At first, the validation of proposed MSM approach is performed by comparing the present results with those reported by Pugno and Ruoff [25] introducing quantized fracture mechanics (QFM). As an energybased theory, QFM modifies continuum-based fracture mechanics by substituting the differentials in Griffith's criterion with finite differences. Fig. 3 represents the stress concentration versus number of omitted atoms. In the QFM results, it is considered that the width of the SLGS tends to infinity $(h \rightarrow \infty)$, however, the MSM results have been depicted for zigzag SLGSs with three finite widths. MSM results reveal that the stress concentration factor is size dependent. One can see that the MSM acts as an upper limit for QFM and when $\mathrm{h}$ increases the difference between MSM and QFM fades away. It confirms that the application of the presented MSM approach is reliable for atomistic evaluation of stress concentration factor of SLGSs with circular holes. It is meaningful to figure out the dependence of stress concentration factor to the chirality, the width and the relative size of the circular hole. Fig. 4 illustrates the variation of stress concentration factor, $\mathrm{Kg}$, versus variation of dimensionless hole size, $\alpha=/ \mathrm{d}$ h, for a series of armchair and zigzag SLGSs. In order to investigate the size effect, the width of SLGSs is varied between the range of $h=10 \mathrm{~nm}$ to $\mathrm{h}=25 \mathrm{~nm}$. It is seen that, similar to the classical stress concentration factor in Eq. (5), increasing the dimensionless parameter of the hole, $\alpha$, causes a rise in stress concentration factor, $\mathrm{Kg}$. It should be noted that due to discrete nature of SLGSs removing a circular region at its center makes an almost circular-shaped hole and therefore a fluctuated ascending trend for $\mathrm{Kg}$ is observed. To enhance the usability of results, a relationship between the stress 
concentration factor and the dimensionless size of hole is derived by means of a curve fitting procedure for either armchair or zigzag SLGSs as follows:

$$
\begin{aligned}
& K_{g}^{\text {Zigzag }}=\left(27.47 \alpha^{3}-22.69 \alpha^{2}+9.19 \alpha+1.12\right)\left(\frac{h}{n m}\right)^{0.08} \\
& K_{g}^{\text {Armchair }}=\left(30.83 \alpha^{3}-26.38 \alpha^{2}+10.08 \alpha+0.8\right)\left(\frac{h}{n m}\right)^{0.19}
\end{aligned}
$$

where $\mathrm{h}$ is the width of SLGS in $\mathrm{nm}$ and consequently the stress concentration factor is size dependent. Eq. (11) and Eq. (12) are used for the evaluation of stress concentration factor in subsequent MSM results. One can generally observe that armchair SLGSs show a larger value of stress concentration than zigzag ones. It means that for a similar load condition they are subjected to higher values of stresses. In the other words the armchair SLGSs with circular holes have lower strength than zigzag ones. This behavior is in agreement with the results reported in the literature which reveals that zigzag SLGSs are stronger against tensile loads than the armchair ones [35]. In armchair configuration, due to the geometrical arrangement of the lattice against the tensile load, the axial forces endured by the equivalent beams are larger than in the zigzag configuration which causes the weaker structural nature of the armchair SLGSs against tension. This is the reason for the larger value of the stress concentration factor in armchair SLGSs. To quantify the chirality effect, the relative increase in the stress concentration due to chirality, $\widetilde{\mathrm{Kg}}$, is defined as:

$$
\widetilde{K}_{g}=\frac{\left(K_{g}^{\text {Ammchair }}-K_{g}^{\text {Zigagag }}\right)}{K_{g}^{\text {Zigzag }}} \times 100
$$

Fig. 5 illustrates the relative increase in stress concentration factor due to armchair configuration versus dimensionless hole parameter, $\alpha$, for three sizes of SLGSs. It is observed that regardless of SLGS size, for circular holes with $\alpha<0.2$, the effect of chirality on stress concentration is rapidly increased and for $0.2<<$ $0 . \alpha 5$ the relative increase in stress concentration is almost constant. However, it should be noticed that the increase in stress concentration factor due to armchair configuration is size dependent and, in the range of presented simulations ( $h=10 \mathrm{~nm}$ to $h=25 \mathrm{~nm}$ ), SLGSs with larger sizes have larger relative increase in stress concentration factor. In Fig. 6, a comparison between the stress concentration factor obtained by MSM and the predicted one by Eq. (5) is depicted to investigate if the classical elasticity is extensible to nano-sized SLGSs. Generally, one can apperceive that for small values of dimensionless hole parameter, $\alpha$, the MSM and classical elasticity results behave completely different so that the classical elasticity start from 3 while the MSM starts around 1.7. It means that for the circular hole whose diameter is small, the application of classical elasticity causes a significant overestimation for both zigzag and armchair configurations. However, for larger values of $\alpha$ the trend of MSM and classical elasticity get closer. By increasing $\alpha$ for zigzag configuration, the classical elasticity and MSM results are almost the same, however, for armchair one the MSM results are significantly larger than those predicted by classical elasticity. Besides, it should be noticed that unlike classical elasticity, the stress concentration factor of nano-sized SLGSs is also size dependent and in the range of the presented simulations ( $h=10 \mathrm{~nm}$ to $h=25 \mathrm{~nm}$ ) by increasing the size of SLGS the stress concentration factor is increased, however, this effect reduces by increasing the width of SLGS. Finally, a comparison between MD and MSM is presented in Fig. 7. Computation of stresses in MD is performed based on viral stress theory [36] which is used in LAMMPS. One can see that the distributions of stresses are similar. It shows that the predicted increasing effect by MSM around circular holes is in good agreement with those predicted by MD simulations. It is noted that due to different view points of stress definition by these two approaches, the values of stresses are not the same, however, the stress concentration factors which reveals the pattern of stress distribution are in good agreement. As a case of comparison, for a zigzag SLGS with $\mathrm{h}=10 \mathrm{~mm}$ and dimensionless parameter of hole, $\alpha=0.5$ the stress concentration factors obtained by MD and MSM are 4.04 and 3.97, respectively. 


\section{Conclusion}

In the present study, the atomistic evaluation of stress concentration factor for SLGSs having circular holes under unidirectional tensile loading is investigated by application of MSM approach and are validated with those obtained by QFM and MD simulations. The conclusions are listed as follows:

- It is confirmed that the application of presented MSM approach is reliable for atomistic evaluation of stress concentration factor of SLGSs with circular holes.

- Increasing the size of circular hole causes an increase in stress concentration factor for both armchair and zigzag configurations.

- For small values of dimensionless hole parameter, $\alpha$, the MSM predicts Kg around 1.7 in contrast to the factor of 3 of elasticity. However, for larger values of $\alpha$, MSM converges to the prediction of classical elasticity.

- Regardless of SLGSs size, stress concentration factor is larger for armchair configuration than zigzag one, which means that armchair SLGSs with circular holes have less strength under tensile loadings.

- For circular holes with $\alpha<0.2$, the increasing effect on stress concentration due to armchair configuration is rapidly raised and for $0.2<<0 . \alpha 5$ it is almost constant.

\section{Acknowledgments}

S.K. Jalali would like to acknowledge the financial support of Kermanshah University of Technology for this research under grant number 1105. N.M.P. was supported by the European Research Council (ERC PoC 2015 SILKENE No. 693670) and by the European Commission H2020 under the Graphene Flagship (WP14 "Polymer composites," No. 696656) and under the FET Proactive (“Neurofibres” No. 732344)

\section{References}

[1] A.C. Ferrari, F. Bonaccorso, V. Fal'ko, et al., Science and technology roadmap for graphene, related twodimensional crystals, and hybrid systems, Nanoscale 7 (2015) 4598-4810.

[2] C. Soldano, A. Mahmood, E. Dujardin, Production, properties and potential of graphene, Carbon 48 (2010) 2127-2150.

[3] R. Ansari, S. Ajori, B. Motevalli, Mechanical properties of defective single-layered graphene sheets via molecular dynamics simulation, Superlattices Microstruct. 51 (2012) 274-289.

[4] B. Mortazavi, S. Ahzi, Thermal conductivity and tensile response of defective graphene: a molecular dynamics study, Carbon 63 (2013) 460-470.

[5] S. Adali, Variational principles for nonlocal continuum model of orthotropic graphene sheets embedded in an elastic medium, Acta Math. Sci. 32 (2012) 325-338.

[6] J.W. Kang, S. Lee, Molecular dynamics study on the bending rigidity of graphene nanoribbons, Comput. Mater. Sci. 74 (2013) 107-113.

[7] M.M. Shokrieh, M. Esmkhani, A.R. Haghighatkhah, Z. Zhao, Flexural fatigue behavior of synthesized graphene/carbon-nanofiber/epoxy hybrid nanocomposites, Mater. Des. 62 (2014) 401-408 (1980-2015). 
[8] R.D. Firouz-Abadi, A.R. Hosseinian, Free vibrations of single-walled carbon nanotubes in the vicinity of a fully constrained graphene sheet, Comput. Mater. Sci. 53 (2012) 12-17.

[9] M. Oubal, S. Picaud, M.-T. Rayez, J.-C. Rayez, Structure and reactivity of carbon multivacancies in graphene, Comput. Theor. Chem. 990 (2012) 159-166.

[10] Z. Ozturk, C. Baykasoglu, M. Kirca, Sandwiched graphene-fullerene composite: a novel 3-D nanostructured material for hydrogen storage, Int. J. Hydrog. Energy 41 (2016) 6403-6411.

[11] Y. Gao, P. Hao, Mechanical properties of monolayer graphene under tensile and compressive loading, Phys. E: Low-Dimens. Syst. Nanostruct. 41 (2009) 1561-1566.

[12] C. Li, T.-W. Chou, A structural mechanics approach for the analysis of carbon nanotubes, Int. J. Solids Struct. 40 (2003) 2487-2499.

[13] A. Sakhaee-Pour, Elastic properties of single-layered graphene sheet, Solid State Commun. 149 (2009) 91-95.

[14] K. Hashemnia, M. Farid, R. Vatankhah, Vibrational analysis of carbon nanotubes and graphene sheets using molecular structural mechanics approach, Comput. Mater. Sci. 47 (2009) 79-85.

[15] A. Sakhaee-Pour, Elastic buckling of single-layered graphene sheet, Comput. Mater. Sci. 45 (2009) 266270.

[16] S.-P. Wang, J.-G. Guo, L.-J. Zhou, Influence of Stone-Wales defects on elastic properties of graphene nanofilms, Phys. E: Low.-Dimens. Syst. Nanostruct. 48 (2013) 29-35.

[17] C.G. Wang, L. Lan, Y.P. Liu, H.F. Tan, X.D. He, Vibration characteristics of wrinkled single-layered graphene sheets, Int. J. Solids Struct. 50 (2013) 1812-1823.

[18] R.D. Firouz-Abadi, H. Moshrefzadeh-Sany, H. Mohammadkhani, M. Sarmadi, A modified molecular structural mechanics model for the buckling analysis of single layer graphene sheet, Solid State Commun. 225 (2016) 12-16.

[19] A. Hashimoto, K. Suenaga, A. Gloter, K. Urita, S. lijima, Direct evidence for atomic defects in graphene layers, Nature 430 (2004) 870-873.

[20] M.P. Ariza, M. Ortiz, Discrete dislocations in graphene, J. Mech. Phys. Solids 58 (2010) 710-734.

[21] J. Zang, S. Ryu, N. Pugno, Q. Wang, Q. Tu, M.J. Buehler, et al., Multifunctionality and control of the crumpling and unfolding of large-area graphene, Nat. Mater. 12 (2013) 321-325.

[22] M.C. Wang, C. Yan, L. Ma, N. Hu, M.W. Chen, Effect of defects on fracture strength of graphene sheets, Comput. Mater. Sci. 54 (2012) 236-239.

[23] S. Yadav, Z. Zhu, C.V. Singh, Defect engineering of graphene for effective hydrogen storage, Int. J. Hydrog. Energy 39 (2014) 4981-4995.

[24] L. Liu, M. Qing, Y. Wang, S. Chen, Defects in graphene: generation, healing, and their effects on the properties of graphene: a review, J. Mater. Sci. Technol. 31 (2015) 599-606.

[25] N.M. Pugno †, R.S. Ruoff $\ddagger$, Quantized fracture mechanics, Philos. Mag. 84 (2004) 2829-2845.

[26] N.M. Pugno, Dynamic quantized fracture mechanics, Int. J. Fract. 140 (2006) 159-168.

[27] N. Pugno, New quantized failure criteria: application to nanotubes and nanowires, Int. J. Fract. 141

(2006) 313-323. 
[28] N.M. Pugno, The role of defects in the design of space elevator cable: from nanotube to megatube, Acta Mater. 55 (2007) 5269-5279.

[29] W.D. Pilkey, D.F. Pilkey, Peterson's Stress Concentration Factors, Wiley, Hoboken, New Jersey, 2008.

[30] A.P. Boresi, R.J. Schmidt, Advanced Mechanics of Material, Wiley, Hoboken, New Jersey, 2003.

[31] S. Bae, H. Kim, Y. Lee, X. Xu, J.S. Park, Y. Zheng, et al., Roll-to-roll production of 30-inch graphene films for transparent electrodes, Nat. Nanotechnol. 5 (2010) 574-578.

[32] V. Palermo, I.A. Kinloch, S. Ligi, N.M. Pugno, Nanoscale mechanics of graphene and graphene oxide in composites: a scientific and technological perspective, Adv. Mater. 28 (2016) 6232-6238.

[33] S.J. Stuart, A.B. Tutein, J.A. Harrison, A reactive potential for hydrocarbons with intermolecular interactions, J. Chem. Phys. 112 (2000) 6472-6486.

[34] W.G. Hoover, Canonical dynamics: equilibrium phase-space distributions, Phys. Rev. A. 31 (1985) 16951697.

[35] Y. Chu, T. Ragab, C. Basaran, The size effect in mechanical properties of finite-sized graphene nanoribbon, Comput. Mater. Sci. 81 (2014) 269-274.

[36] A.K. Subramaniyan, C.T. Sun, Continuum interpretation of virial stress in molecular simulations, Int. J. Solids Struct. 45 (14-15) (2008) 4340-4346. 


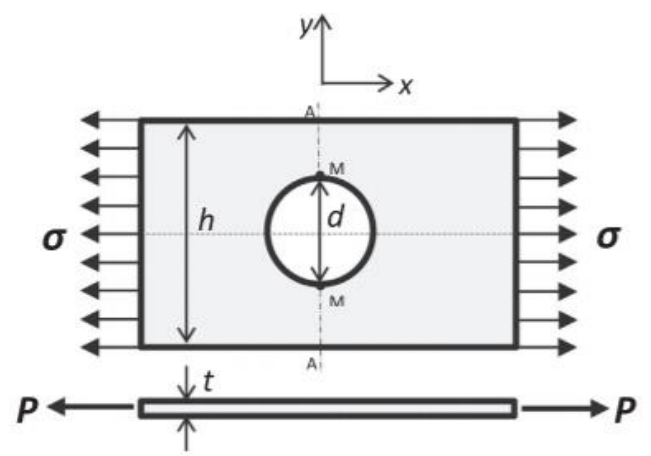

Fig. 1. A thin panel of finite width having a single circular hole subjected to uniform uniaxial tension.

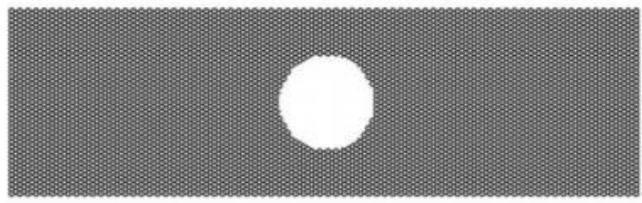

(a)

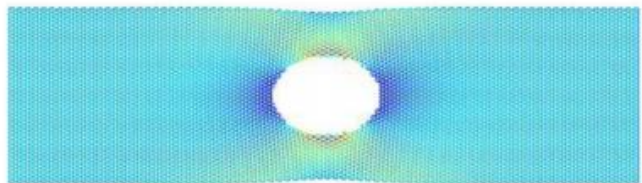

(b)

Fig. 2. (a) MSM frame-like beam model of SLGSs having circular holes. (b) An example of stress distribution obtained by MSM which is used for investigating the stress concentration factor.

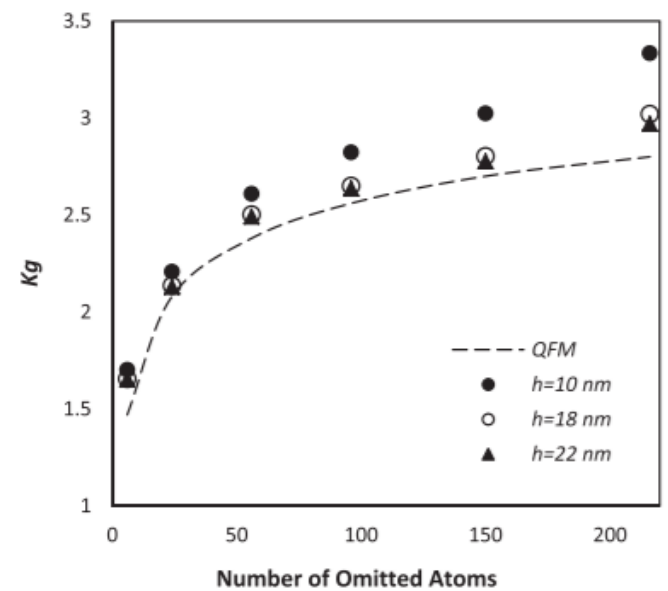

Fig. 3. Comparison between stress concentration factors obtained by MSM for finite $h$ and QFM in the limit of $h \rightarrow \infty$. 


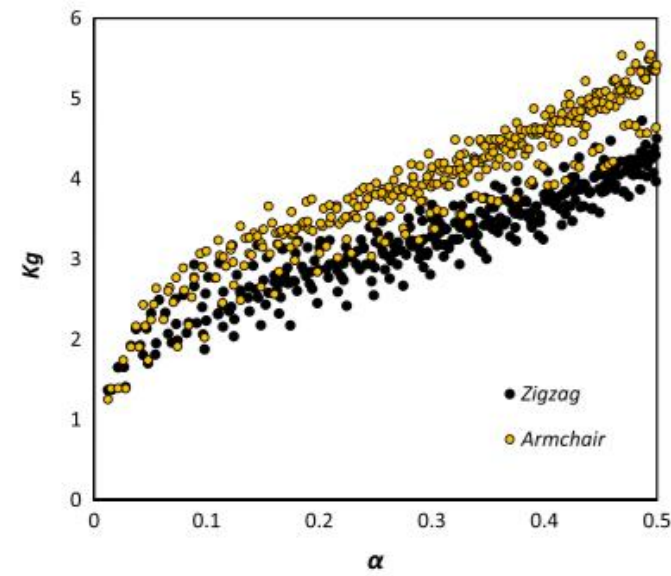

Fig. 4. Stress concentration factors evaluated by MSM for both zigzag and armehair SLGSs of various widths.

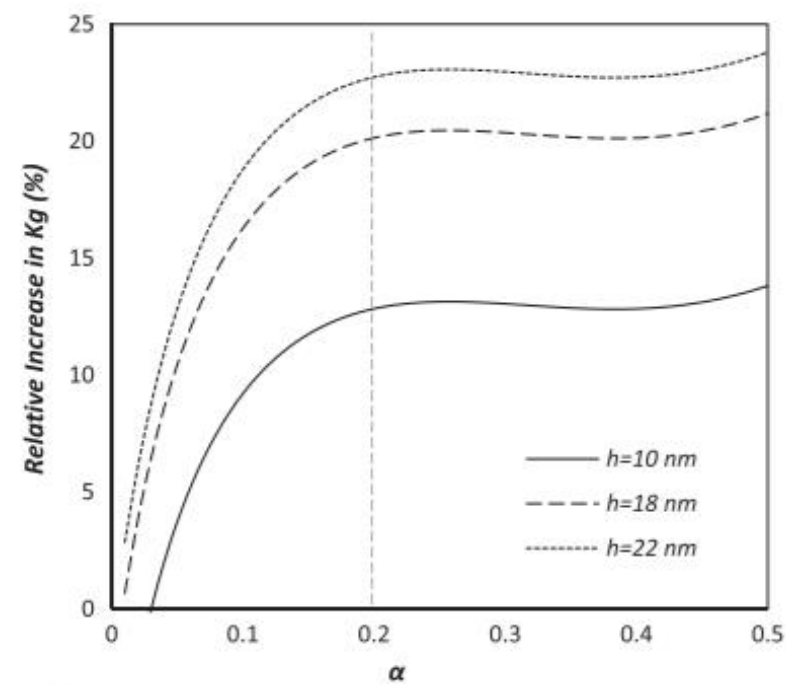

Fig. 5. Relative increase in stress concentration due to chirality effect.

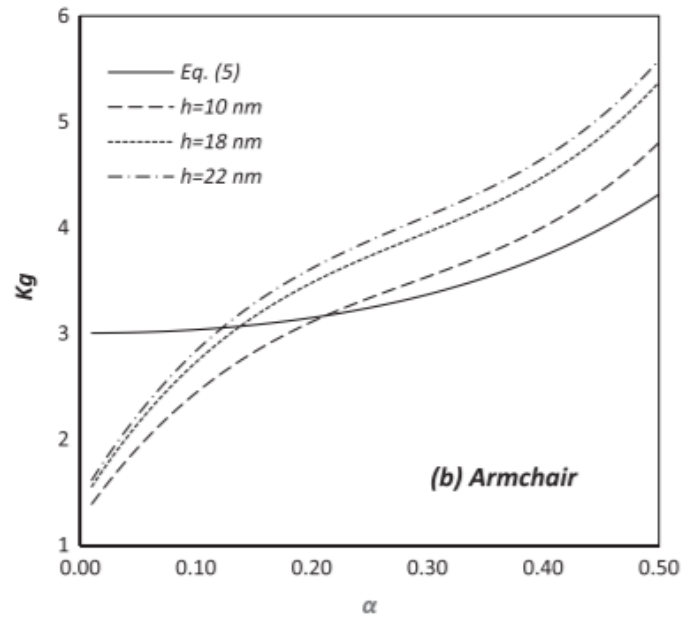

Fig. 6. Comparison between stress concentration factors of SLGSs predicted by classical elasticity theory and obtained by MSM approach for (a) zigzag, (b) armchair. 
(a)
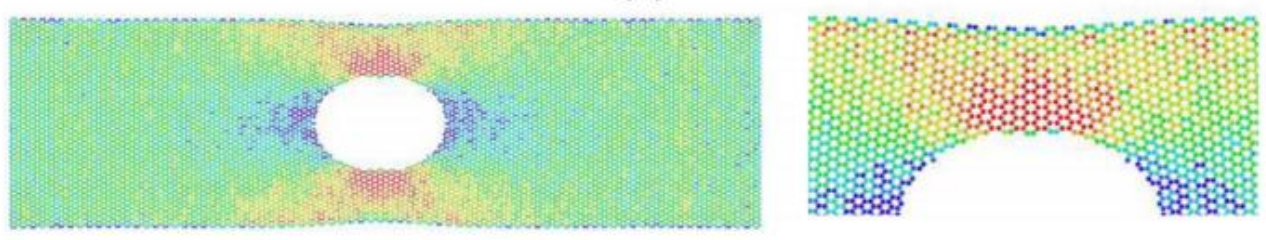

(b)

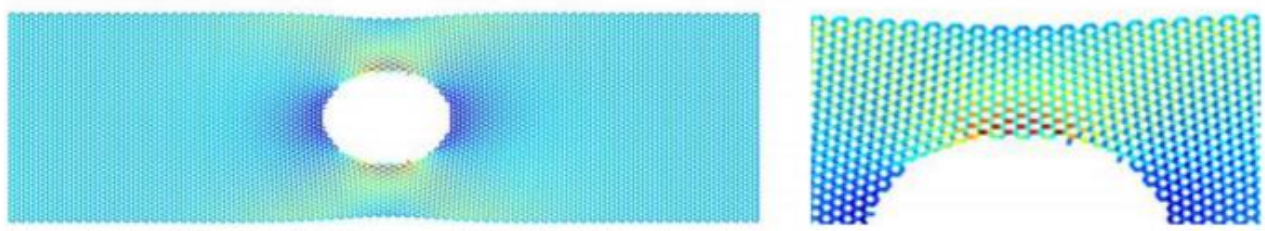

Fig. 7. The stress distribution contours in SLGSs having circular holes. (a) MD simulation, (b) MSM approach. 